\title{
Avaliação da dor lombar crônica em praticantes de musculação: estudo clínico, controlado, randomizado e duplo-cego
}

Evaluation of chronic lumbar pain in

musculation practice: a clinical, controlled, randomized, double blind study
FisiSenectus . Unochapecó Ano 5, n. 2 - Jul/Dez. 2017 p. $38-46$

Lucas Amaro Salsa Ferreira. lucasamaro9@hotmail.com

Fisioterapeuta, Centro Universitário de Itajubá (Fepi) - Itajubá/MG, Brasil.

Danillo Barbosa.danillo.barbosa@hotmail.com

Fisioterapeuta. Mestre em Engenharia Biomédica pela Universidade do Vale do Paraíba (UNIVAP). Doutor em Engenharia Biomédica pela Universidade Camilo Castelo Branco. Docente da Universidade Estadual do Centro-Oeste do Paraná (Unicentro), Guarapuava/PR, Brasil.

Ivo I. Kerppers. ivo@ig.com.br

Fisioterapeuta. Mestre em Engenharia Biomédica pela Universidade do Vale do Paraíba (UNIVAP). Doutor em Engenharia Biomédica pela

Universidade Brasil (UNIVBRASIL). Docente da Universidade Estadual do Centro-Oeste do Paraná (Unicentro) Guarapuava/PR, Brasil.

Gabriela Xavier. gab_xavier@hotmail.com

Graduanda em Fisioterapia, Universidade Federal de Alfenas (Unifal), Alfenas/MG, Brasil.

Mônica Beatriz Ferreira.monica.ferreira@unis.edu.br

Fisioterapeuta. Mestre em Gerontologia pela Universidade Estadual de Campinas (Unicamp). Coordenadora e Docente do Centro Universitário do Sul de Minas Gerais (Unis), Varginha/MG, Brasil.

\section{Resumo}

Objetivo: 0 objetivo deste estudo foi realizar uma análise da incidência de dor lombar em fisiculturistas. Materiais e Métodos: Trata-se de um ensaio duplo-cego, randomizado e controlado. Este estudo incluiu 300 fisiculturistas, matriculados a, pelo menos, seis meses e treinando pelo menos duas vezes por semana. A amostra foi dividida em dois grupos: homens $(n=150)$ e mulheres $(n=150)$. Os dois grupos foram avaliados, levando em conta dois instrumentos para análise de dor: índice de incapacidade de oswestry e questionário sobre problemas de dor nas costas do Quebec. Resultados: Os resultados da avaliação do índice de incapacidade de oswestry no grupo masculino encontraram um índice significativo, correspondente à incapacidade moderada em $\mathrm{n}=64$ indivíduos deste grupo. A média geral e o desvio padrão de todas as respostas foram $44,26 \pm 178,680$. Os resultados da avaliação do questionário sobre problemas de dor nas costas de Quebec foram expressos como média e desvio padrão. Notou-se que, no grupo masculino do item 19, obteve um índice expressivo de respostas correspondente à nota $88 \pm 32,0$. No grupo de mulheres, observou-se um índice significativo de $79 \pm 19.00$ respostas no item 5 . Conclusão: Conclui-se que a amostra analisada no presente estudo apresenta alguma disfunção lombar devido à prática constante de musculação. Os achados relatados neste estudo estão de acordo com estudos de referência, publicados nos últimos anos,

\section{Fisiß̊nectus}


e sugerem uma avaliação cuidadosa da prática de musculação para não gerar lesões importantes na coluna vertebral, especialmente no segmento lombar.

\title{
Palavras-chave
}

Dor lombar; avaliação da coluna lombar; musculação.

\begin{abstract}
Objective: The objective of this study was to perform an analysis of the incidence of low back pain in bodybuilders. Materials and methods: It is a double-blind, randomized, controlled trial. This study includes 300 bodybuilders, enrolled at least six months and training at least twice a week. The sample was divided into two groups: men $(n=150)$ and women $(n=150)$, the two groups were oriented, taking into account two instruments for pain analysis: oswestry disability index and questionnaire on back pain in Quebec. Results: The results of the evaluation of the developmental disability index are not related to the male group. The overall mean and standard deviation of all responses were $44.26 \pm 178.680$. The results of the evaluation of the questionnaire on the problems of back pain in Quebec were expressed as mean and standard deviation. It was noted that, in the male group of item 19, it obtained an expressive index of responses corresponding to the score $88 \pm 32.0$. There was no group of women, a significant index of $79 \pm 19.00$ responses without item 5. Conclusion: It was concluded that the sample analyzed in this study does not present any lumbar dysfunction due to the constant practice of bodybuilding. The findings reported in the study are in line with landmark studies published in recent years and suggest a careful assessment of bodybuilding practice not to generate important spinal injuries, especially without lumbar segment.
\end{abstract}

\section{Keywords}

Low back pain; lumbar spine evaluation; bodybuilding

\section{Introdução}

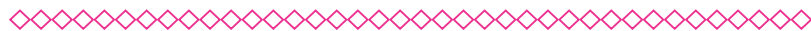

A dor lombar (LBP) é persistente e na maioria dos casos costuma ser crônica, geralmente a dor está localizada na lombar. Os sintomas podem ser físicos e emocionais. Estudos atuais descreveram que entre 15 a $27 \%$ da população pode sofrer de dor lombar ou aresentar os sintomas doIorosos. É importante enfatizar que a dor lombar crônica é caracterizada principalmente pela duração de sintomas dolorosos e incapacidade física superior a 3 três meses contínuos ${ }^{1}$. Estima-se que na América do Norte os custos totais para o tratamento da dor lombar crônica ultrapassam U\$ 600 bilhões ${ }^{2,3}$.

A musculação é uma prática muito antiga. Sugere-se que os primeiros indícios da modalidade de levantamento de peso surgiram na Grécia e no Egito por volta de 4.500 anos a. C. A atividade de musculação ganhou adeptos em todo o mundo e hoje vários benefícios estão relacionados à sua prática, como aumento da força e da massa muscular, resistência muscular, redução da gordura corporal, melhoria da estética da auto-estima, da saúde e da qualidade de vida de seus praticantes ${ }^{4,5}$.

As academias de musculação são cada vez mais procuradas por indivíduos de ambos os sexos, das mais variadas faixas etárias e classes socioeconômicas, mas cada um tem objetivo específico ${ }^{5}$.

Como atualmente, as pessoas estão buscando maneiras de melhorar suas condições de saúde, tanto físicas como psicossociais, uma das estratégias mais comuns é procurar uma academia de musculação; no entanto, nesse local estão expostas a lesões resultantes do exercício, bem como à exacerbação de problemas posturais e musculares existentes ${ }^{6}$.

Os fatores de risco para a dor lombar variam de acordo com a população estudada e podem ser influenciados por: idade, tipo de atividade do trabalho, níveis de estresse psicológico e prática de atividades esportivas. Estima-se que aproximadamente $80 \%$ da população mundial apresentará 
pelo menos um episódio de dor lombar incapacitante ${ }^{7}$.

Destaca-se que qualquer exercício, quando executado incorretamente ou mal prescrito, pode levar a lesões musculoesqueléticas e também ao aumento da pressão no disco vertebral, especialmente na região lombar baixa. Isso pode, inclusive, contribuir para uma hérnia de disco no futuro ${ }^{8}$.

0 objetivo deste estudo foi realizar uma análise sobre a incidência de dor lombar em praticantes de musculação, ponderada por instrumentos de avaliação validados em todo o mundo para esse finalidade.

\section{Materiais e métodos}

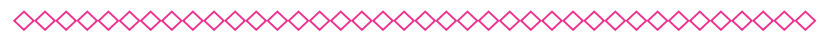

\section{Tipo de Estudo}

É um estudo clínico, controlado, aleatorizado, duplo-cego, desenvolvido no Departamento de Fisioterapia do Centro Universitário de Itajubá (FEPI), sob a coordenação da disciplina de fisioterapia traumato-ortopédica.

\section{Amostra}

Foram convidados para o estudo 300 fisiculturistas, de três academias de musculação diferentes, e que estavam matriculados a pelo menos seis meses, e que tinham uma frequência mínima de duas vezes por semana. A amostra consistiu de sujeitos de ambos os sexos, 150 homens e 150 mulheres. A Tabela 1 descreve as características gerais da amostra.

\section{Seleção da Amostra, Randomização e Alocação}

A seleção dos participantes baseou-se em um convite global feito a três centros de musculação localizados na região central da cidade de Itajubá, em Minas Gerais, Brasil. 0 convite foi estendido a todos os membros da academia, respeitando os critérios de inclusão. Todos os participantes receberam o termo de consentimento, com explicações claras e objetivas sobre o estudo. Dessa forma, todos os participantes elegíveis para participarem do estudo assinaram o termo de consentimento livre e esclarecido, o qual está de acordo com as
Diretrizes e Normas Regulamentadoras de Pesquisas Envolvendo Seres Humanos e Comitê de Ética do FEPI e foi entregue em duas vias (uma para o pesquisado e outra para os pesquisadores).

A amostra inicial consistiu de 380 participantes, de ambos os sexos, que estavam regulamente matriculados nas academias. Após analisar, avaliar, e realizar as entrevistas em todos os sujeitos da amostra, levando em consideração os critérios de inclusão e exclusão, a amostra final ficou com 300 indivíduos incluídos e 80 sujeitos excluídos.

A amostra foi dividida em dois grupos, conforme apresentado na Tabela 1: masculino $(n=150)$ e feminino $(n=150)$. Ambos os grupos foram avaliados, levando em consideração dois instrumentos para análise de dor: Instrumento de Incapacidade de Dor Lombar de Queceb (QBPQ) ${ }^{9}$ e Índice de Incapacidade de Oswestry (ODI) 9 . 0 Quadro 1 traz a Amostra Universal.

\section{Critério de inclusão}

Os participantes do estudo foram selecionados de acordo com os seguintes critérios de inclusão: praticar musculação por pelo menos três meses, ter uma frequência mínima de treinamento de dois dias por semana, não estar sendo submetido à terapia medicamentosa, não apresentar sintomas de dor na coluna vertebral lombar nos últimos três meses, não têm diagnóstico de doença da coluna lombar, ter idade entre 18 e 60 anos, aceitar participar de todas as fases do estudo e entregar o termo de consentimento livre e esclarecido assinado.

\section{Critérios de exclusão}

Os participantes neste estudo foram excluídos de acordo com os seguintes critérios: não praticavam musculação por pelo menos três meses, não tinham frequência mínima de treinamento de dois dias por semana, estavam sendo submetidos a alguma terapia medicamentosa, apresentavam sintomas como dor na coluna lombar nos últimos três meses, havia algum diagnóstico de doença na coIuna lombar, não ter idade entre 18 e 60 anos, não participar de todas as fases do estudo e não assinar e ou não entregar o termo de consentimento livre e esclarecido assinado. 
Procedimentos de Coleta de Dados e Cegamento

A coleta de dados foi iniciada após um sorteio com dez academias de musculação na cidade de Itajubá, em Minas Gerais, após a seleção, três academias foram escolhidas para participar do estudo. Inicialmente, um convite foi feito para todos os alunos regularmente matriculados nas academias. Em seguida, uma análise foi realizada nas três academias para conhecer o número total de interessados em participar do estudo. Foi realizado um sorteio aleatório, considerando o número de inscrição do aluno na academia. Ao final, a amostra contou com 300 indivíduos incluídos e 80 sujeitos excluídos.

A coleta de dados foi realizada apenas pelo pesquisador do estudo, visto que possui experiência clínica na área de fisioterapia esportiva, fisioterapia traumático-ortopédica e fisioterapia reumatológica. Este pesquisador recebeu apenas a informação do local, hora e data do início da coleta. As demais informações foram restritas.

As coletas foram planejadas da seguinte forma: academia A, academia B e academia C. As três academias totalizaram 300 participantes, sendo assim o avaliador iniciou a coleta em uma academia predeterminada, escolhida por sorteio. As coletas foram realizadas individualmente em uma sala reservada, onde foram aplicados dois instrumentos de avaliação: $\mathrm{QBPQ}^{9}$ e $\mathrm{ODI}^{9}$. Depois que os sujeitos responderam aos dois instrumentos, eles foram nomeados apenas pelas iniciais do nome, bem como a academia de origem, como D.K.N (academia A).

\section{Instrumentos de avaliação}

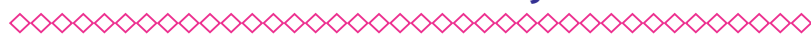

\section{Instrumento ODI 9}

O Índice de Incapacidade de Oswestry é usado para avaliar a função da coluna lombar, incluindo a dor e as variáveis de atividade física ${ }^{10}$. Este instrumento contém dez perguntas, com seis alternativas, variando de 0 a 5 . A primeira questão avalia a intensidade da dor e as outras nove avaliam o efeito da dor nas atividades diárias, como cuidados pessoais (vestir e tomar banho), levantar pesos, andando, sentado, de pé, dormindo, na sua vida sexual, social e em locomoção.

A pontuação total é dividida pelo número de perguntas respondidas, multiplicado pelo número 5 . 0 ponto de corte da validação brasileira de ODI para dor lombar é de aproximadamente 4,45 pontos $(63,2 \%$ de sensibilidade e $81,8 \%$ de especificidade), representando o mínimo sinal clínico importante para essa diferença. Assim, a avaliação final segue esta fórmula: ([score $\div$ (sem perguntas respondidas $\times 5)] \times 100)^{11}$.

\section{Instrumento $\mathrm{QBPQ}^{9}$}

Este instrumento de avaliação específica para dor lombar consiste em 20 itens que descrevem a dificuldade de realizar atividade física leve. Está dividido em seis domínios: descansar e dormir, sentar-se e ficar de pé, andar, movimentos, curvar-se e parar, e objetos pesados.

Cada item possui uma escala com seis pontuações (0-5), sendo 0 a ausência de dificuldades e 5 a incapacidade máxima para realizar a atividade. Assim, a pontuação final varia de 0 a 100. 0 valor mínimo de variação na pontuação para uma alteração clínica é de 15 a $20^{12}$.

\section{Análise de Dados}

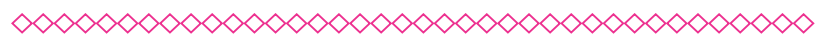

Os dados coletados foram submetidos inicialmente à análise estatística descritiva, com tabelas de frequência para variáveis categóricas e medidas de posição e dispersão para variáveis contínuas detalhadas em Excel $^{\circledR}$. Em seguida, os dados foram determinados em média e desvio padrão de todas as variáveis do estudo.

\section{Resultados}

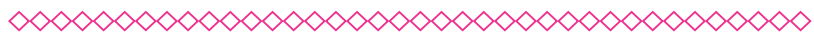

Os resultados da avaliação do ODI) ${ }^{9}$ (Tabela 2.0) no grupo masculino encontraram um índice significativo, correspondente à incapacidade moderada em $n=64$ indivíduos desse grupo. Todas as variáveis foram expostas em média e desvio padrão, correspondendo a 44,26 $\pm 178,680$. 
A Tabela 2 apresenta a distribuição dos sujeitos de acordo com os resultados obtidos no ODI.

Os resultados da avaliação do QBPQ) ${ }^{9}$ foram expostos (Tabela 3) como média e desvio padrão. Notou-se que, no grupo masculino do item 19 referente 0 instrumento de avaliação "transportar dois sacos de compras", obteve um índice expressivo de respostas correspondente à nota $88 \pm 32,0$. Os itens 18 (pontuação $71 \pm 19,80$ ) e 20 (pontuação 66 $\pm 19,00$ ) também obtiveram valores significativos. No grupo de mulheres observado no item 5, corresponde ao questionamento: durante 20-30 minutos obteve um índice importante de compromissos (\#79 á 19,00) e itens 11 (\#\#pontu-

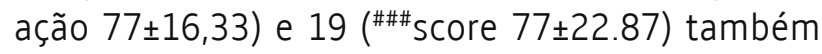
recebeu altas pontuações.

\section{Discussão}

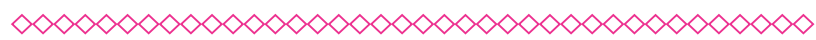

A coluna vertebral é o eixo principal do corpo humano, e está constantemente sendo submetida a forças diversas que agem sobre ela, podendo gerar agravos devido a mudanças posturais que movem o centro de gravidade do corpo, comprometendo uma estrutura óssea esquelética. 0 desalinhamento postural está presente entre $60 \%$ a $80 \%$ na população, especialmente em adultos jovens em sua fase produtiva ${ }^{13}$.

Embora os benefícios que provêm da prática de exercícios físicos, especialmente a musculação, sejam um fator extremamente importante e que não pode ser esquecido ou simplesmente ignorado pelos profissionais, possiveis lesões podem ser geradas ou agravadas devido à pratica de exercícios de resistência ${ }^{14}$.

No presente estudo, os resultados mostraram que ambos os grupos (feminino e masculino) apresentaram valores significativos para os índices de dor e incapacidade, avaliados pelos instrumentos $\mathrm{ODI}^{9}$ e $\mathrm{QBPQ}^{9}$.

Um estudo ${ }^{15}$ envolvendo profissionais que trabalham com musculação, contou com 138 sujeitos, com relatos de dor musculoesquelética, com idades variando de 18 a 60 anos, de ambos os sexos. Na análise utilizando o $\mathrm{ODI}^{9}$, os participantes apresentaram uma média de 28,12, com $56,5 \%$ dos indivíduos com "deficiência moderada", 28,2\% com "incapacidade mínima" e 15,3\% com "deficiência severa".

Um estudo ${ }^{16}$ que usou o ODI ${ }^{9}$, envolvendo 110 indivíduos, com relatos de dor lombar, mostrou os seguintes resultados: incapacidade mínima de $79 \%$, incapacidade moderada $11 \%$ da amostra ${ }^{16}$.

Em outro estudo ${ }^{17}$, envolvendo 30 pacientes provenientes de um serviço de saúde pública no Brasil e 30 pacientes com características biológicas similares provenientes do hospital universitário de Salamanca, verificou-se que todos relataram dor nas costas e histórico de atividade de musculação. Os resultados foram: 24,2 $\pm 15,2(16,5-31,9)$ para mulheres brasileiras e $24,7 \pm 10,9(15,9-33,5)$ para mulheres espanholas e $37,7 \pm 14,6(29,7-$ $45,7)$ mulheres, para homens brasileiros e 30,4 $\pm 21,1(21,8-38,9)$ Homens espanhóis. Houve, também, uma comparação da dor lombar entre os dois países, com os resultados de 24,4 $\pm 13,3$ (19,4$29,4)$ para brasileiros e $34,3 \pm 18(27,5-41)$ para espanhóis e um significado de $t=-2,411 ; P=0,019$, um estudo em que os resultados corroboram com o presente estudo.

0 presente estudo corrobora os estudos mencionados na literatura. E os métodos de avaliação e aplicação também podem ser usados da forma quantitativa, que correlaciona a quantidade de questões relevantes para quantificar a presença de dor ou deficiência provocada pela dor lombar, mas também pode ser aplicada como forma comparativa.

\section{Conclusão}

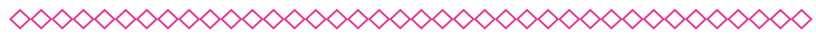

Conclui-se que a amostra analisada no presente estudo apresenta dor ou alguma disfunção lombar devido à prática constante de musculação. Os achados analisados neste estudo estão de acordo com estudos de referência, publicados nos últimos anos, que descrevem sobre a importância de realizar uma avaliação cuidadosa para a prática de musculação. Eventualmente, sinais e sintomas agudos também devem ser observados para não contribuir para formações de patologias mais graves. Sugere-se que mais estudos sejam realizados nesta linha, a fim de orientar os atletas praticantes 
de exercícios resistidos (musculação), fisiculturistas e outros.

\section{Referências}

$\infty \times \infty \times \infty \times \infty \times \infty \times \infty \times \infty \times \infty \times \infty \times \infty \times \infty)$

1. Mariano TY, Urman RD, Hutchison CA, Jamison RN, Edwards RR. Cognitive Behavioral Therapy (CBT) for Subacute Low Back Pain: a Systematic Review. Curr Pain Headache Rep. 2018 Feb 23; 22(3): 15 .

2. Gaskin DJ, Richard P. The economic costs of pain in the United States. J Pain. 2012; 13(8):71524.

3. Hoy D, Brooks P, Blyth F, Buchbinder R. The epidemiology of low back pain. Best Pract Res Clin Rheumatol. 2010; 24(6):769-81.

4. Hallal PC, Andersen LB, Bull FC, Guthold R, Haskell W, Ekelund U. Global physical activity levels: surveillance progress, pitfalls and prospects. Lancet. 2012; 380(9838): p. 247-57.

5. Souza GL, Moreira NB, Campos W. Ocorrência e características de lesões entre praticantes de musculação. Revista Saúde e Pesquisa. 2015; 8(3): 469-477.

6. Castro AAM, Guerino RPZ, Ferreira TK, Portes $L A$, Porto EF. Percepção de lesões musculares em praticantes de musculação em academias com e sem supervisão de fisioterapeuta: uma análise custo-efetividade. Life Style Journal. 2015; 2(1):11-22.

7. Gotfry AO, Filho ESV, Viola DC, Lenza M, Silva JA, Emi AS, et al. , Análise epidemiológica, de hábitos de vida e de fatores psicossociais de pacientes com dorso lombalgia em unidade de pronto atendimento ortopédico. Einstein. 2015; 13(2):243-8.

8. Gusmão TMR, Ribeiro KLS, Granja KSB, Sant'Ana HGF, Machado AP. Desempenho funcional do exercício de agachamento; Ciências Biológicas e da Saúde; Maceió. 2015; 2(3):45-56.
9. Falavigna, A Teles AR, Braga GL, Barazzetti DO, Lazzaretti L, Tregnago Instruments of Clinical and Function Evaluation Spine Surgery. Coluna/ Columna. 2011; 10(1):62-7.

10. Vigatto R, Alexandre NM, Correa Filho HR. Development of a Brazilian Portuguese version of the Oswestry Disability Index: cross-cultural adaptation, reliability, and validity. Spine. 2007; 32(4):481-6.

11. Coelho RA, Siqueira FB, Ferreira PH, Ferreira ML. Responsiveness of the Brazilian-Portuguese version of the Oswestry Disability Index in subjects with low back pain. Eur Spine J. 2008; 17(8):1101-6.

12. Doleys DM, Klapow JC, Hammer M. Psychological evaluation in spinal cord stimulation therapy. Pain Rev. 1997; 4:189-207.

13. Leite AAAS, Santosa LS, Araújo MO, Neto JLC. Dor lombar e exercício físico: uma revisão sistemática. Revista Baiana de Saúde Pública. 2015; 39(2): 442-459.

14. Souza GL, Moreira NB, Campos W. Ocorrência e características de lesões entre praticantes de musculação. Revista Saúde e Pesquisa. 2015; 8(3):469-477.

15. Vieira A, Nonnenmacher LFQ, Bartza PT, Bueno AF, Macedo DS. Perfil de usuários com dores musculoesqueléticas crônicas encaminhados ao "grupo da coluna". Revista Baiana de Saúde Pública. 2014; 38(3):571-584.

16. Oliveira GD, Casa Júnior AJ. Prevalência de Iombalgia e avaliação da capacidade funcional lombar em praticantes de musculação; estudos, Goiânia. 2014; 41(2):247-258.

17. Souza DPR, De-La-Peñas CF, Vellejo FJM, Blanco JFB, Gutiérrrez LM, Sendín F A. Differences in pain perception, health-related quality of life, disability, mood, and sleep between Brazilian and Spanish people with chronic non-specific low back pain. Braz. J. Phys. Ther. 2016; 1(7):33-9. 


\section{Anexos}

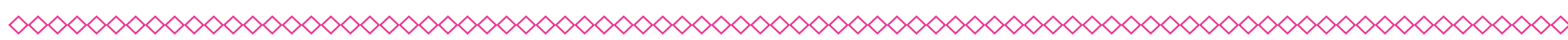

Tabela 1 - Características da Amostra

\begin{tabular}{lccccc}
\hline Variável & Amostra & Idade (Anos) & Peso (kg) & Altura (Mts) & $\begin{array}{c}\text { Tempo de } \\
\text { Musculação (M) }\end{array}$ \\
Masculino & $\mathrm{N}=150$ & $\pm 28,5$ & $\pm 82,4$ & $\pm 1,81$ & $\pm 18,3$ \\
Feminino & $\mathrm{N}=150$ & $\pm 26,3$ & $\pm 67,6$ & \pm 1.62 & $\pm 15,2$ \\
Média & - & $\pm 27,4$ & $\pm 75,0$ & $\pm 1,715$ & $\pm 16,75$ \\
\hline Desvio Padrão & - & $\pm 1,555635$ & $\pm 10,39447$ & $\pm 0,13435$ & $\pm 2,192031$ \\
\hline
\end{tabular}

Nota: Os dados foram expressos em média depois de serem tabulados no Excel ${ }^{\circledR}$.

(clique para voltar ao texto)

Quadro 1 - Amostra Universal

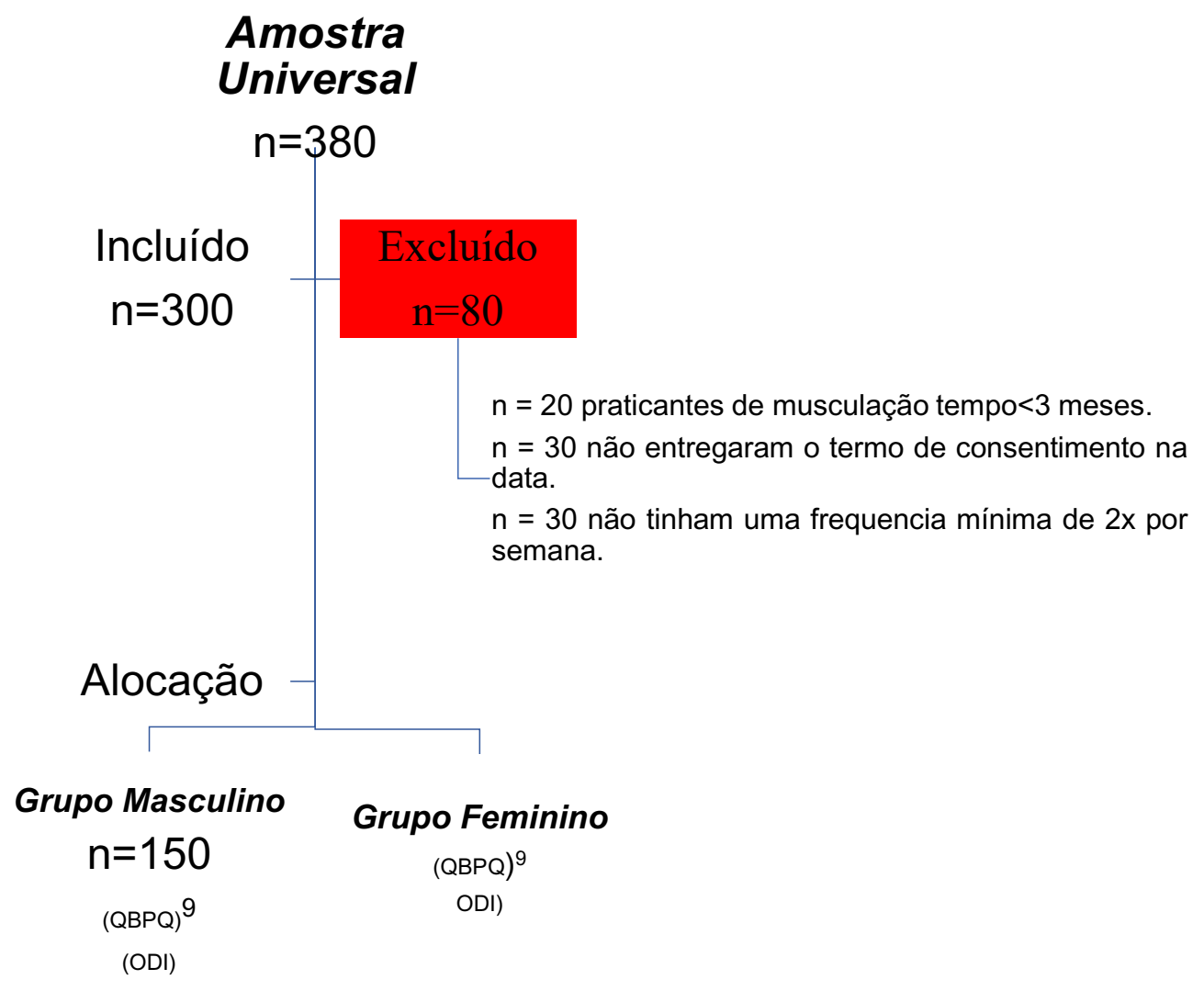

(clique para voltar ao texto) 
Tabela 2 - Distribuição dos sujeitos de acordo com os resultados obtidos no ODI.

\begin{tabular}{|c|c|c|}
\hline Resultados (ODI) ${ }^{9}$ & N= 150 (Grupo Masculino) & N= 150 (Grupo Feminino) \\
\hline Incapacidade Mínima & $\mathrm{n}=55$ & $*^{*} \mathrm{n}=68$ \\
\hline Incapacidade Moderada & ${ }^{*} \mathrm{n}=64$ & $\mathrm{n}=56$ \\
\hline Incapacidade Severa & $\mathrm{n}=30$ & $\mathrm{n}=26$ \\
\hline Aleijado (Deficiência) & $\mathrm{n}=1$ & $\mathrm{n}=0$ \\
\hline Inválido & $\mathrm{n}=0$ & $\mathrm{n}=0$ \\
\hline Média e Desvio Padrão & $44,26 \pm 178,680$ & $38,33 \pm 166,880$ \\
\hline
\end{tabular}

Nota: Este instrumento contém 10 seções, com seis alternativas, variando de 0 a 5 . Para cada seção de seis instruções, 0 ponto total é 5 . Se a primeira resposta for verificada, o ponto é 0 . Se for o último, o ponto é 5 . As respostas intermediárias são pontuadas de acordo com esta classificação. 0 cálculo básico para analisar as dez seções do estudo obedece a esta fórmula: ([pontuação $\div$ (sem perguntas respondidas $\times 5)] \times 100)^{11}$. A interpretação dos dados é feita levando em consideração os seguintes indicadores: $0 \%$ a $20 \%$ - incapacidade mínima; $21 \%$ a $40 \%$ - incapacidade moderada; $41 \%$ a $60 \%$ - incapacidade grave; $61 \%$ a $80 \%$ - aleijado; $81 \%$ a $100 \%$ - inválido. Tendo em conta uma situação pós-operatória, deve adotar os seguintes critérios: \% a $20 \%$ - excelente; $21 \%$ a $40 \%$ - bom; $41 \%$ a $60 \%$ - inalterado; > 60\% - piora. Os resultados foram expostos na Tabela 2, e foram expressos separadamente no grupo masculino e no grupo feminino. Os resultados foram expressos como média e desvio padrão. 
Tabela 3 - Distribuição dos sujeitos de acordo com os resultados obtidos no QBPQ ${ }^{9}$

\begin{tabular}{ccc}
\hline QBPQ $)^{9}$ & $\begin{array}{c}\text { N= } 150 \text { (Groupo Masculino) } \\
\text { Média/Desvio Padrão }\end{array}$ & $\begin{array}{c}\text { N= } 150 \text { (Grupo Feminino) } \\
\text { Média/Desvio Padrão }\end{array}$ \\
1 & Ponto $44 \pm 12,90$ & Ponto $39 \pm 09,44$ \\
2 & Ponto $39 \pm 09,80$ & Ponto $29 \pm 11,34$ \\
3 & Ponto $29 \pm 09,50$ & Ponto $22 \pm 10,11$ \\
4 & Ponto $29 \pm 10,90$ & Ponto $20 \pm 05,22$ \\
5 & Ponto $77 \pm 22,00$ & \#Ponto $79 \pm 19,00$ \\
6 & Ponto $33 \pm 12,80$ & Ponto $44 \pm 17,00$ \\
7 & Ponto $19 \pm 17,80$ & Ponto $21 \pm 06,22$ \\
8 & Ponto $19 \pm 09,40$ & Ponto $16 \pm 04,77$ \\
9 & Ponto $31 \pm 17,90$ & Ponto $44 \pm 12,99$ \\
10 & Ponto $44 \pm 18,90$ & Ponto $66 \pm 19,33$ \\
11 & Ponto $55 \pm 17,70$ & \#\#Ponto $77 \pm 16,33$ \\
12 & Ponto $33 \pm 09,33$ & Ponto $28 \pm 09,99$ \\
13 & Ponto $17 \pm 6,90$ & Ponto $13 \pm 04,50$ \\
14 & Ponto $15 \pm 22,0$ & Ponto 09 $\pm 02,88$ \\
15 & Ponto $33 \pm 11,0$ & Ponto $41 \pm 09,00$ \\
16 & Ponto $55 \pm 19,0$ & Ponto $66 \pm 22,33$ \\
17 & Ponto $44 \pm 22,9$ & Ponto $55 \pm 21,99$ \\
18 & ** Ponto $71 \pm 19,8$ & Ponto $67 \pm 17,28$ \\
19 & * Ponto $88 \pm 32,0$ & \#\#\# Ponto $77 \pm 22,87$ \\
20 & *** Ponto 66 $\pm 19,0$ & Ponto $55 \pm 23,99$ \\
\hline
\end{tabular}

Nota: Este instrumento é dividido em domínios e contém 20 perguntas, onde cada resposta pode ser pontuada entre 0 e 5. Quando a nota atribuída é 0 , significa que não há dificuldade; quando a nota é 5 , significa dificuldade e impotência máxima para realizar a atividade proposta pelo pesquisador. A pontuação final varia de 0 a 100 pontos, o que significa pior condição clínica quando a pontuação é maior que 33. 0 escore entre 15 e 20 pontos é considerado o valor mínimo de variação na avaliação deste instrumento. No grupo masculino, observaram-se variações significativas na média e desvio padrão de algumas das questões do instrumento. No item 19, referente à questão: "carregar duas bolsas de compras"', obteve a maior pontuação mostrando uma insatisfação significativo entre homens *pontuação 88ะ32,0, vale a pena

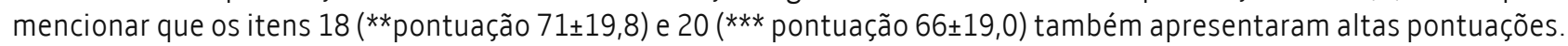
0 grupo de mulheres apontou no item $5(\# 79 \pm 19,00)$, referindo-se à pergunta: "ficar por 20 a 30 minutos" obedecendo a maior pontuação e indicando dificuldade em realizar essa tarefa, os itens 11 e 19 obtidos respectivamente \#\#core 77士 16,33 e \#\#\# pontuação $77 \pm 22,87.4 .0$. 